\title{
Local identification of the stress-strain curves of metals at a high strain rate using repeated micro-impact testing
}

\author{
G. Kermouche ${ }^{a, *}$, F. Grange ${ }^{b}$, C. Langlade ${ }^{c}$ \\ a Ecole des Mines de Saint-Etienne, SMS Department, LGF Lab UMR CNRS 5307, 158 Cours Fauriel, 42023 Saint-Etienne Cedex 2, France \\ ${ }^{\mathrm{b}}$ Université de Lyon, Ecole Nationale d'Ingénieurs de Saint-Etienne, Laboratoire de Tribologie et Dynamique des Systèmes, UMR 5513 CNRS/ECL/ENISE, \\ 58 Rue J. Parot, 42023 Saint-Etienne Cedex 2, France \\ ${ }^{\mathrm{c}}$ LERMPS Lab., EA 3316, Université de Technologie de Belfort-Montbéliard, 90010 Belfort, France
}

Understanding the effect of various mechanical processes such as shot peening requires the knowledge of metal behavior at a high strain rate. The identification of this behavior is often performed using Hopkinson's bar devices. However, the resulting stress-strain curves correspond to a bulk behavior of the material and thus do not take into account the modification induced by surface preparations or surface treatments. In this paper, we have proposed a method based on local micro-impact testing to identify the stress-strain curves of metals near their surface. More precisely, this method is based on the determination of the best stress-strain curve which allows to reproduce the growth of the residual imprint at each impact for a given impact energy. The advantage of the method developed in this paper lies in its simplicity and low cost. In the first part, the repeated impact set-up is presented. Then the FEM strategy is detailed and the identification method developed. In the last part of this paper, an application to AISI1045 and AISI316L steels highlights the great interest of this method to obtain better stress-strain curves than those classically used. It points out the need to identify appropriate surface stress-strain curves when the surface behavior is concerned. As a matter of fact it should also concern other manufacturing processes such as machining or finishing.

\section{Introduction}

Using efficient finite element simulations, it is now possible to gain a thorough understanding of many industrial processes based on impacts such as stamping, turning or milling [1]. Some of these processes are dedicated to the creation of new surfaces and others are used as mechanical surface treatments to improve near-surface properties. The most famous process of this kind is shot peening. During the treatment, the sample is peened by a large number of shots over a short period of time, which creates a compressive residual stress area in the sub-surface. It has been shown in numerous papers how finite element analyses may be used to explain the creation of such residual stress fields [2]. It appears that one key-parameter is the stress-strain curve of the materials treated. Indeed, the choice of the stress-strain curve will directly influence the level and size of the induced compressive residual stress field. One of the main difficulties is that shot peening induces high strain rates (higher than $100 \mathrm{~s}^{-1}$ ) and it is well known that the stress-strain relation of most metals at such

\footnotetext{
* Corresponding author. Tel.:+33 4774200 74; fax: +33477420157.

E-mail address: kermouche@emse.fr (G. Kermouche).
}

strain rates is radically different from the stress-strain curve at lower strain rates. The identification of the stress-strain curves at the strain rates induced by the peening process is then required to correctly model the shot peening treatment.

Identifying metal behavior at high strain rates is often performed by means of Hopkinson's bar devices [3]. However, the resulting stress-strain curve corresponds to a bulk behavior of the material and thus does not take into account the modification induced by surface preparations or surface treatments. To correctly describe or model the mechanical response of engineering surfaces submitted to peening or scratch processes [4], this local mechanical behavior has to be known. It is the reason why another kind of mechanical testing has to be used. Generally, local mechanical properties are extracted using the nano-indentation technique [5-7]. However, the standards of nano-indentation are not really adapted to the identification of metal behavior at high strain rates. Specific dynamic indentation devices - instrumented nano-impacts - have been designed [8-11], which permit such measurements. However, the instrumentation of such devices is really difficult and expensive, which limits their practical use.

In this paper a new method based on multiple impacts in a same point is developed to extract the elastoplastic behavior of metals at strain rates close to those induced by shot peening 
processes. It is based on the use of a standard industrial micropercussion device, which allows to accurately control the locations of impacts and their kinetic energies $[12,13]$. It is to be noted that no specific additional instrumentation is required to use the method developed in this paper. Here the main objective is not to identify the true stress-strain curve for different strain rates but to obtain a fairly accurate mechanical behavior of the surface so as to describe the modification induced by shot peening processes correctly. The strategy developed in this paper is to determine the best stress-strain curve which allows to reproduce the growth of the residual imprint at each impact for a given impact energy.

In the first part, the repeated impact set-up is presented. Then the FEM strategy is detailed. Finally the identification method is developed and an application of this method to an AISI1045 steel and an AISI316L stainless steel is presented.

\section{Indentation versus impact}

The impact of spherical balls under normal incidence was well described by Tabor [14] in the case of dynamic hardness measurements. When dynamic effects can be neglected, except on the indenter kinematics [15], an impact under normal incidence can be considered to be a classical indentation. The main difference is that impacts are energy-controlled whereas classical indentation loadings are load-controlled or displacement-controlled. For low impact energies, only elastic deformation takes place. In this case, the relation between impact energy, geometrical and mechanical parameters has been derived by Johnson [15] based on Hertz theory of elastic indentation. For higher impact energy, the plastic deformation of metal occurs until the kinetic energy has been consumed. Finally, there is a release of elastic stresses in the indenter and the material and a permanent impression is visible (Fig. 2). The same results are observed when elastic and plastic deformations occur under indentation loadings.

In the theory of indentation [16], it is possible to define equivalent values of the stress and strain fields. It is obvious that stress, strain and strain rate fields are not uniform all over the deformed area. In fact, these equivalent parameters correspond to an average level of the mechanical fields [17,7]. The representative stress is related to the mean pressure, which is often called hardness. According to Tabor, the representative stress and strain are linked by the stress-strain curve of the indented materials. For instance, the spherical indentation representative strain [17-19] is often written

$\varepsilon=0.2 \frac{a}{R}$

where $a$ is the residual imprint radius after indentation under a given load and $R$ is the ball radius. Hence the stress-strain curve of metals can be determined very easily using different indentation loads and by measuring the residual imprint radius after each indentation. Although this identification method was strongly criticized in the past $[20,16,21]$, it points out the fact that it is possible to determine a closed-form of the material stress-strain curve using simple spherical indentation experiments.

Let us now consider the representative strain rate. Subhash et al. [9] proposed to define the nominal strain rate as the ratio of the indenter speed over the imprint radius. Mok and Duffy [22] and Tirupataiah and Sundararajan [23] proposed to define the nominal strain rate as the ratio of the nominal strain over the impact duration, the nominal strain being defined according to Tabor's definition [17]. It is clear that these definitions are only an overall estimate but they make it possible to measure qualitatively the strain rate level induced by an impact. Here, we propose to adopt the simple definition of Tabor

$\dot{\varepsilon}=\gamma \frac{v}{a}$

where $v$ is the impact velocity, $a$ the residual imprint radius and $\gamma$ a constant (set to 1 in the present paper). Let us note that this definition is in very good agreement with previous results on the indentation of time-dependent materials [7]. Taking these different relations into consideration, impacting can be considered to be a classical spherical indentation but with a much higher level of strain rate.

Because impacts are energy-controlled, it is not really possible to apply the classical methods developed for spherical indentation experiments. In this paper we propose a new method based on repeated impacts with constant kinetic energy. Kermouche et al. [24] have shown that two impact regimes can be identified. The transient impact regime is characterized by a growth of the residual imprint at each impact [12] and an increase of the maximum impact load. This transient impact regime is followed by the stabilized impact regime characterized by the shakedown of the structure to a macroscopic elastic response. During this regime there is no more increase of the contact area per impact.

The main idea of the method developed here is to use the transient impact regime. According to the spherical indentation theory, the increase of the residual imprint and of the maximum load per impact is related to the stress-strain curve of the impacted material. If these measurements could be performed with sufficient accuracy, then a perfect control of the impact energy would not be required. However, if the measure of the post-mortem imprint morphology can be performed with very high accuracy, the determination of the maximum impact loading has to be taken with caution. Indeed, this could be a very difficult task with regard to the dynamic response of the impact tester and also to the load sensor capability. To overcome this difficulty and because it is possible to accurately control the impact energy, the method presented in this paper is only based on the measure of the residual imprint morphology at each impact for a given impact energy.

\section{Experimental set-up}

The principle of the repeated impact device used in this study has already been presented in previous papers [12] and is sketched in Fig. 1. A rigid indenter, ended by a hemispherical tip electromagnetically accelerated is pushed onto the sample surface under normal incidence. Zirconia balls $(E=200 \mathrm{GPa}$,

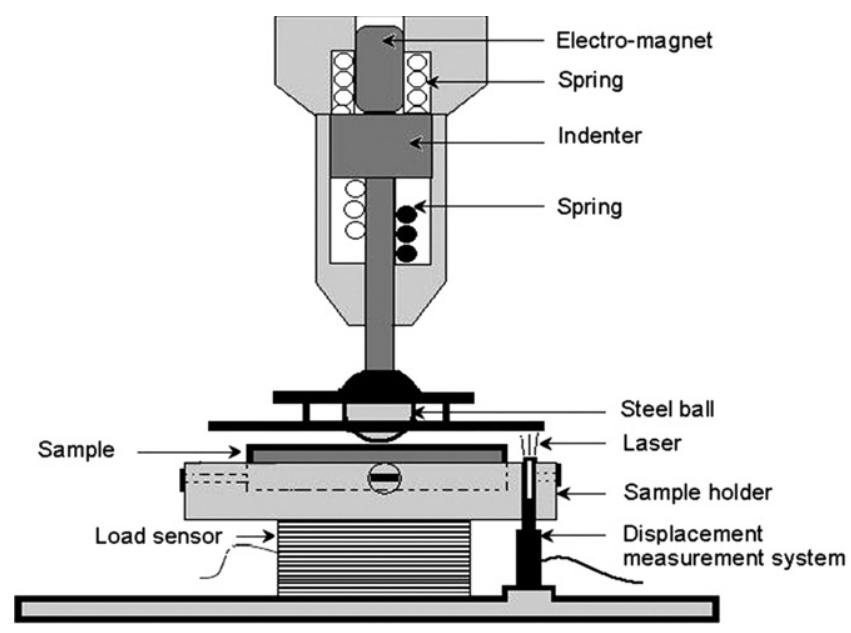

Fig. 1. The principle of the impact testing device [12]. 


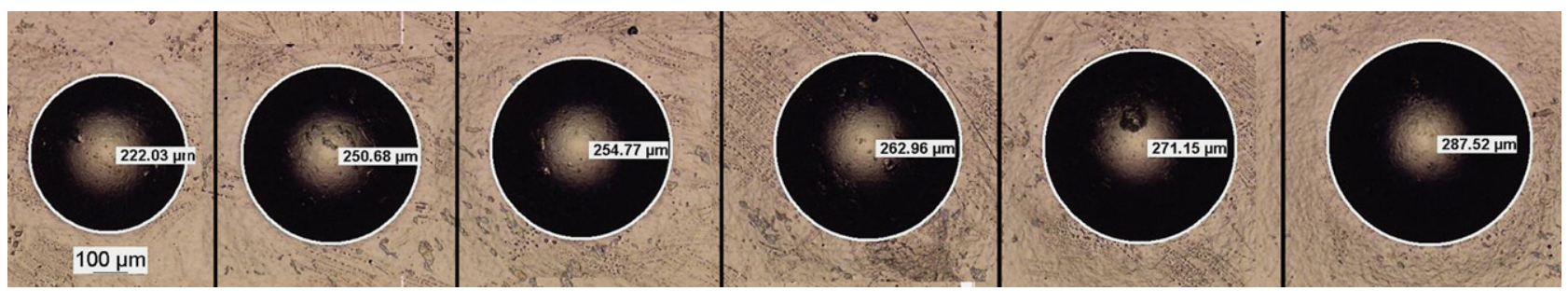

Fig. 2. Residual imprints on an AISI1045 steel for 1, 2, 3, 5, 7 and 10 impacts for an impact energy of $8 \mathrm{~mJ}$.

hardness: $800 \mathrm{Hv}$ ) with a diameter of $2 \mathrm{~mm}$ (grade 10) have been used as impacting tips leading to a total indenter mass of $174 \mathrm{~g}$. A constant acceleration being generated by the electromagnets, the indenter velocity and kinetic energy just before the impact may be directly determined using the indenter weight and its initial position above the sample surface. The incident kinetic energy was checked using a laser diode displacement sensor. The usual impact energy range belongs to $[1,21] \mathrm{mJ}$, which corresponds to an impact speed range of $[50-500] \mathrm{mm} / \mathrm{s}$. For most metals tested with this impact device, the imprint radius belongs to [100-400] $\mu \mathrm{m}$. Hence, according to Eq. (2), the range of impactinduced strain rates belongs to $[100,1000] \mathrm{s}^{-1}$. For most metals, this range is sufficiently limited to consider that an equivalent time-independent stress-strain curve is accurate enough to model the mechanical response of the surface.

As explained in the previous section, the strategy of the method developed in this paper is based on the measure of the growth of the residual imprint morphology - depth and radius with the number of impacts. To illustrate this, Figs. 2 and 3 show the growths of the residual imprints induced by repeated impacts on an AISI1045 steel $(200 \mathrm{Hv})$ for an impact energy of $8 \mathrm{~mJ}$.

Because the impact tester is not perfectly rigid, a significant part of the kinetic energy may be lost in the device deformation according to Eq. (3)

$T=W_{\text {contact }}+W_{\text {device }}$

where $T$ is the impact energy, $W_{\text {contact }}$ is the work used to deform the sample and the tip, and $W_{\text {device }}$ is the work lost in the device. This last component has been estimated from impacts on a Tungsten Carbide sample ( $E=650 \mathrm{GPa}, H=1300 \mathrm{Hv}$ ) using different impact energies. Indeed, impacts being perfectly elastic on such materials (no residual imprint after each impact condition used), $W_{\text {contact }}$ can be calculated from the maximum load measured using Hertz contact theory. $W_{\text {device }}$ is then obtained from Eq. (3). Let us note that here $W_{\text {device }}$ is assumed to depend on the maximum impact load only.

\section{FEM modeling strategy}

Most authors use explicit finite element analyses to model the behavior of materials under impact loadings [25-27]. Indeed explicit finite element analyses are well adapted to fast nonlinear dynamic problems. However, the time step in such analyses has to be small enough to maintain the stability of the solution procedure. The time step is related to the density, the elastic modulus and the mesh length. The smaller the mesh length of the smallest element, the smaller the time step. Consequently accurate simulations are very costly and may sometimes lead to instable results [28]. In order to reduce the computation time and the accuracy of the parametric study, a static analysis using an energy equivalence has been adopted to model normal impact loadings [24]. A quasi-static approach can be used to model impact loading if and only if there is no evidence of dynamic effects, except on the indenter kinematics [15]. This hypothesis

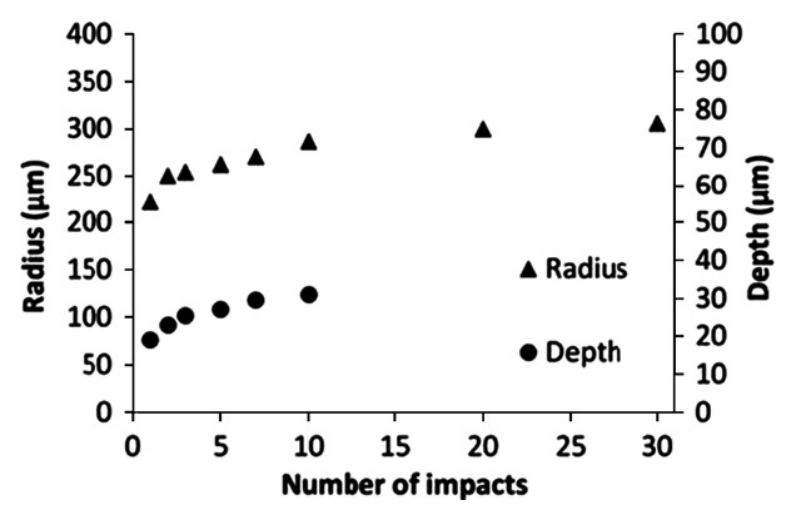

Fig. 3. Growth of the residual imprint radius (depth) on an AISI1045 steel with the number of impacts for an impact energy of $8 \mathrm{~mJ}$.

is valid if the impact time is much higher than the elastic wave propagation in the contact region [29]. This last condition can be written

$t_{\text {impact }}>\frac{2 a}{c}$

where $t_{\text {impact }}$ is the impact time, a is the maximum contact radius and $c$ the longitudinal elastic wave speed in the sample. In our experiment the contact duration is about $200 \mu \mathrm{s}$ whereas the ratio $2 a / c$ is about $100 \mathrm{~ns}$. According to this theoretical result, a quasi-static model can be used instead of a dynamic model. However, the development of a reverse analysis requires the use of the most accurate model. For that purpose, Appendix A shows that the results obtained with the quasi-static finite element model are in very good agreement with those resulting from classical dynamic explicit finite element calculations.

\subsection{Quasi-static simulation}

The quasi-static simulation is based on standard indentation models. Calculations have been performed with Systus/Sysweld [30] using axisymmetric elements and a large displacement/large strain option (updated Lagrangian formulation). The mesh is particularly refined near the contact zone, but also sufficiently wide to approximate a semi-infinite solid (Fig. 4). In order to ensure plastic incompressibility, four node quadrilateral isoparametric elements with a selective reduced integration scheme are used in the plastically deformed area. The plastic flow is described via a plastic von Mises stress. The loading is achieved by imposing a quasi-static displacement of the indenter as explained above. For each impact, the penetration depth of the ball is stopped when the impact energy $T$ equals the sum of $W_{\text {contact }}$ and $W_{\text {device. }}$. The contact between the indenter and the work-piece has been assumed to be frictionless. It is shown in Appendix B that the sensitivity of the residual imprint morphology to the friction conditions is very weak. The parametric study has been performed assuming a perfectly elastic indenter with the properties of Zirconia ( $E=200,000$ and $v=0.3$ ). 


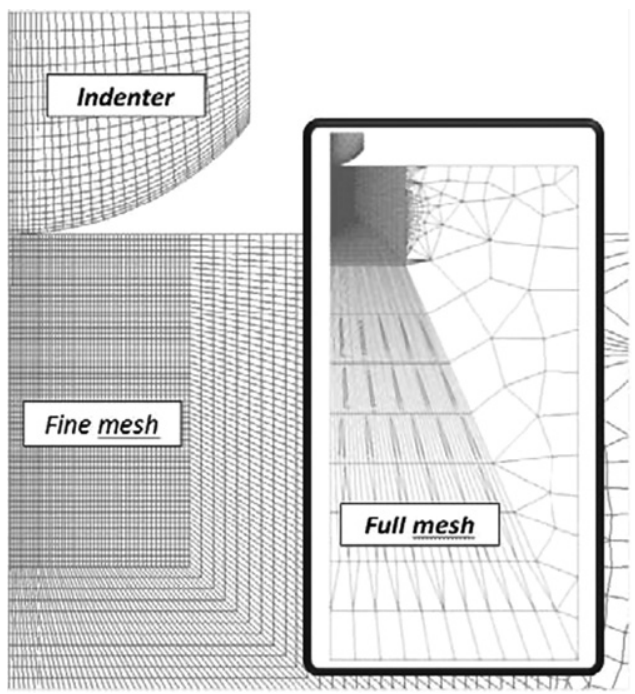

Fig. 4. Finite element mesh used for modeling the quasi-static simulation.

\section{Development of the identification method}

\subsection{Material model}

To simplify finite element investigations on indentation, many authors $[31,32,16]$ proposed to use Hollomon's law to describe the stress-strain curve of metals. This is a two-parameter power law description $\sigma=k \varepsilon^{n}$ [31] where $k$ is a strength coefficient and $n$ the strain hardening exponent. It has been shown that for many pure and alloyed engineering materials, it gives a good approximation of the uniaxial stress-strain curve. It is to be noted that the stress sensitivity to the strain rate is not taken into account in Hollomon's law. According to Eq. (2), the nominal strain rate induced by the impact device belongs to the range [100-1000 s $\left.\mathrm{s}^{-1}\right]$. It is considered here that this strain rate range is sufficiently limited to assume that a time-independent stress-strain curve such as Hollomon's law will accurately describe the mechanical response of materials to such impact loadings. This hypothesis is valid for a large class of metals. Let us also note that this law is only a good approximation of most metal stress-strain curves but it cannot be used to model phenomena related to kinematic hardening and more specifically to cyclic loading. Collin et al. [33] have shown that the use of combined isotropic and kinematic hardening allows to better reproduce cyclic spherical indentation curves. However, it appears that kinematic hardening does not have a first-order effect on the indentation curve. Consequently, for a first approach, the kinematic hardening has not been considered in the present study.

\subsection{Parametric study}

The strategy developed in this paper is to determine the best stress-strain curve which allows to reproduce the growth of the residual imprint at each impact for a given impact energy. In order to cover a large range of stress-strain curves and impact conditions, an extensive parametric study has been performed. The input parameters of the finite element analysis are

- $K=[500,1000,1500,2000,2500,3000,4000,5000] \mathrm{MPa}$,

- $n=[0.0,0.1,0.2,0.3,0.4,0.5]$,

- $E_{\text {impact }}=[2,5,8,15,21] \mathrm{mJ}$.

The elastic properties of the impacted materials have been fixed to $E=200 \mathrm{GPa}$ and $v=0.3$. For each finite element calculation, 10

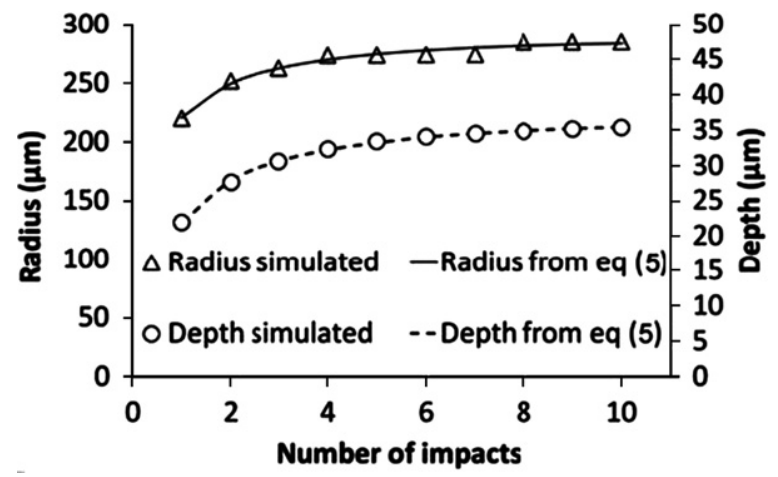

Fig. 5. Imprint depth and radius versus number of impacts simulated and calculated from Eq. (5) $\left(k=2000 ; n=0.2\right.$ and $\left.E_{\text {impact }}=8 \mathrm{~mJ}\right)$.

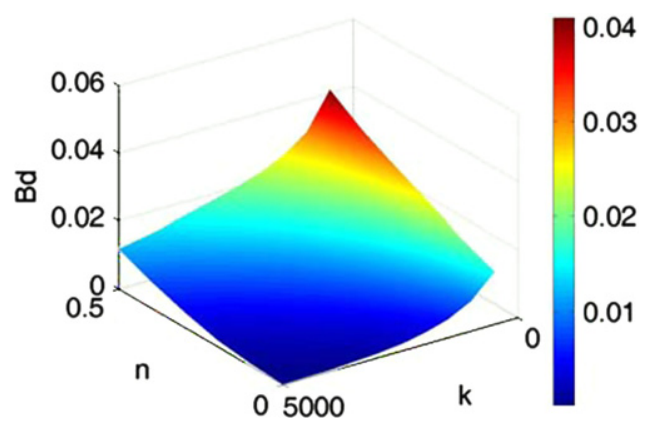

Fig. 6. Parameter $B d$ of Eq. (5).

loading/unloading cycles were simulated, which corresponds to 10 repeated impacts. For each numerical simulation corresponding to a given set of $\left[k, n, E_{\text {impact }}\right]$, the growths of depth and radius with the number of impacts are obtained as shown in Fig. 5. It appears that these variations can be expressed as

$x(N)=A_{x} \log (N)^{2}+B_{x} \log (N)+C_{x}$

where $N$ is the number of impacts, $x$ is a geometrical parameter of the residual imprint, i.e. radius $r$ and/or depth $d$. Let us note that the choice of Eq. (5) was drawn from a large number of studies on indentation testing $[31,32,16] . A_{x}, B_{x}, C_{x}$ are functions of $\left[k, n, E_{\text {impact }}\right]$ and have been stored in two databases: one for the growth of depth $\left(A_{d}, B_{d}, C_{d}\right)$ and the other for the growth of radius $\left(A_{r}, B_{r}, C_{r}\right)$. To illustrate this, function $B_{d}(K, n)$ corresponding to an energy of $8 \mathrm{~mJ}$ is plotted in Fig. 6.

\subsection{Identification method}

Similarly to numerical results, experimental results can be approximated by Eq. (5). Let us denote $r e(N)$ and $\operatorname{de}(N)$ the experimental values of the residual imprint radius and depth after $N$ impacts at a given energy. For the impact energy investigated, the optimal values of $K$ and $n$ are determined by finding the minimum of the following functions:

$I_{x}(k, n)=100 * \frac{\sqrt{\int_{0}^{N \max }(x-x e)^{2} d x}}{\sqrt{\int_{0}^{N \max }(x)^{2} d x}}$

where $x$ is a geometrical parameter of the residual imprint, i.e. radius $r$ and/or depth $d$. This function could be related to the general concept of variance, or standard deviation, and it has been drawn from the classical least square method. The minimization of the function $I_{r}(k, n)$ (resp $\left.I_{d}(k, n)\right)$ makes it possible to identify the best couple $(K, n)$ which permits to adjust the experimental variation of the radius (resp depth) to the number of impacts. The minimization of these two functions may lead to different results, 


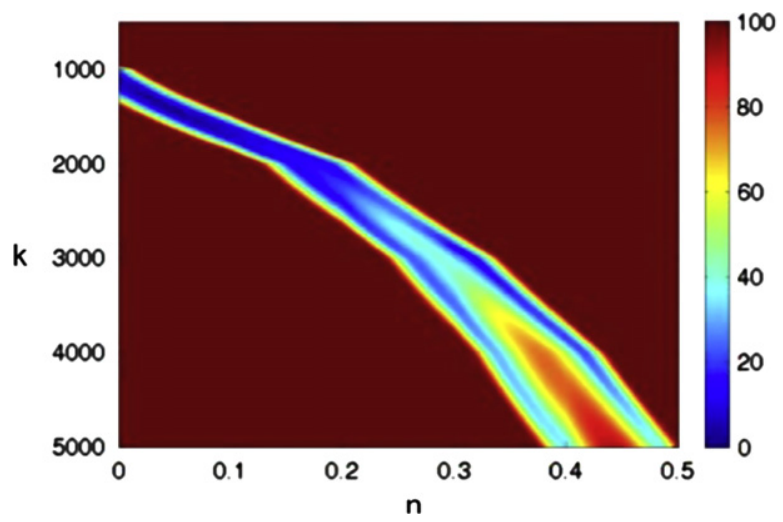

Fig. 7. Application of the method using the data of impacts with an energy level of $8 \mathrm{~mJ}$ on the AISI1045 steel.

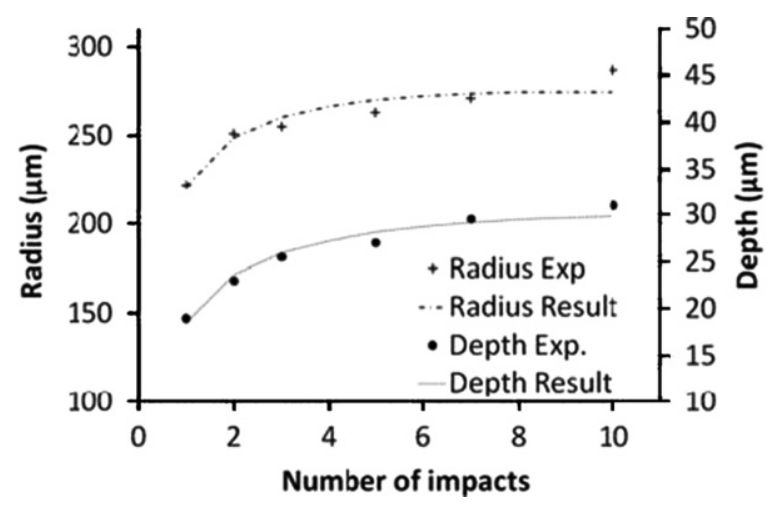

Fig. 8. Radius and depth of the residual imprint obtained with the experimental tests and the results of the method.

thus making it difficult to determine which one is better. For that purpose, we propose to minimize the product of these two functions $I(k, n)$, which should lead to another solution satisfying both experimental variations of radius and depth. For instance, the function $I(k, n)$ is plotted in Fig. 7 for ten impacts at $8 \mathrm{~mJ}$ on the AISI1045. The minimization of this function leads to the couple $k=1225 \mathrm{MPa}$ and $n=0.026$. Fig. 8 illustrates the good agreement between the growths of radius and depth measured experimentally and those resulting from this couple $(K, n)$.

\subsection{Set of solution}

The remaining difficulty of reverse analysis is the solution unicity. The minimization method used here leads to a single couple $(K, n)$ solution. However, a set of couples $(K, n)$ which gives a value close to the global minimum can be identified. It is obvious, that the couple $(K, n)$ solution will strongly depend on the experimental uncertainties related to the measure of imprint radius and depth and also on the energy loss due to the experimental device stiffness. Hence the couple $(K, n)$ identified by the present inverse method is to be taken with caution. Let us have a look at the stress-strain curves resulting from different couples $(k, n)$ of the set identified above (Fig. 9). It appears that these stress-strain curves are very close on a given strain range. The upper limit is very close to the spherical indentation representative strain given in Eq. (1).

Therefore, it indicates that the stress-strain curve identified with the inverse method is only available on a given strain range according to ball indentation theory. For instance, the strain interval from Fig. 2 for AISI1045 is 0.04-0.06 referring to Eq. (1). In order to identify a wider strain range, it is thus necessary to run

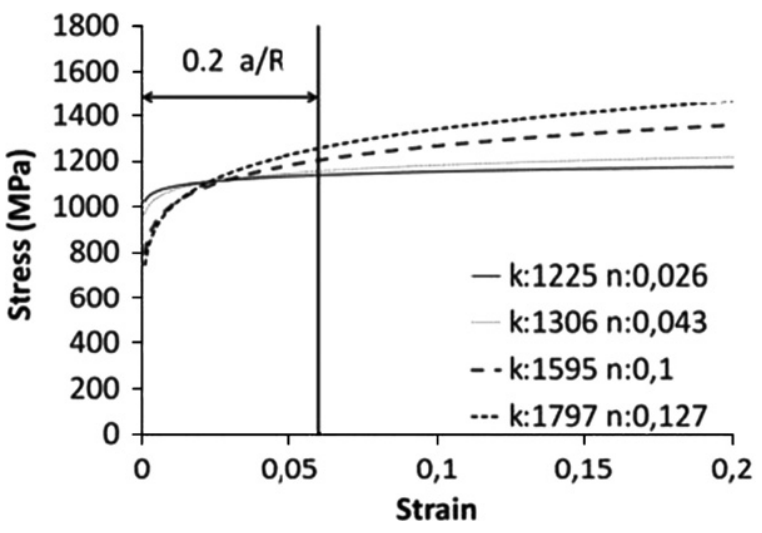

Fig. 9. Stress-strain curves corresponding to the set of couples $(K, n)$ which gives a value close to the global minimum. Global minimization leads to the couple $k=1225 \mathrm{MPa}$ and $n=0.026$. It appears that these stress-strain curves are very close on the strain range corresponding to the nominal strain induced by spherical indentation: $\varepsilon=0.2 a / R$.

the identification method using different impact energy to assess the behaviour at smaller and/or larger strains.

\section{Applications}

\subsection{Materials}

Repeated impact tests have been conducted on an annealed AISI1045 steel (200 HV) and an AISI316L stainless steel (150 Hv). The surface was mechanically polished with abrasive papers up to 1200 grit size. Surface finishing was achieved using diamond paste up to $3 \mu \mathrm{m}$ grit before ultrasonic cleaning in ethanol. Two impact energies $(8 \mathrm{~mJ}$ and $21 \mathrm{~mJ}$ ) were used for each material. According to Eq. (2), the nominal strain rate induced by these impacts belongs to the range $\left[100 \mathrm{~s}^{-1} ; 1000 \mathrm{~s}^{-1}\right]$. According to literature data $[3,34]$, the stress difference for a strain of 0.1 within this strain rate range is lower than $30 \mathrm{MPa}$ for the AISI1045 steel and lower than $60 \mathrm{MPa}$ for the AISI316L.

For each residual imprint, the depth and radius have been measured using a 3D optical profilometer and an optical microscope. Before applying the present method, it is important to discuss the problem of the temperature rise during the impact which could induce adiabatic deformation. Indeed such a phenomenon is not taken into account in finite element simulations, therefore it can alter the results of the inverse identification. Consequently it is important to state about the conditions for which the adiabatic deformation can be neglected. For that purpose, Johnson [15] proposed the following non-dimensional parameter for measuring the behavior regime for the impact of metals

$\Gamma=\frac{T}{3 Y_{d} R^{3}}$

where $T$ is the impact energy, $Y_{d}$ is the dynamic yield stress and $R$ is the indenter radius. Between $\Gamma=10^{-3} \mathrm{~s}^{-1}$ and $10^{-1} \mathrm{~s}^{-1}$, the impact can be reasonably described by the quasi-static indentation theory and heating effects are thus negligible. In the present study, $\Gamma$ is always lower than $10^{-2} \mathrm{~s}^{-1}$ for the two metals tested (with $Y_{d}=800 \mathrm{MPa}$ ). Therefore, the identification procedure can be applied as such.

\subsection{Application to the AISI1045 steel}

The impact energies used here are $8 \mathrm{~mJ}$ and $21 \mathrm{~mJ}$. For each impact energy level, a stress-strain curve is deduced using the 
identification method developed above. These curves are plotted in Fig. 10. Let us note that the stress-strain curves identified with these two impact energy levels are very close, which points out the reliability of the method and the fact that the difference in strain rate between $8 \mathrm{~mJ}$ impacts and $21 \mathrm{~mJ}$ impacts is not significant for this steel. We propose here to define the stress-strain curve of the AISI1045 steel as the one which minimizes the distance between these two stress-strain curves. Here we obtain the following solution: $k=1344 \mathrm{MPa}$ and $n=0.047$. This curve is also plotted in Fig. 10 . To check this result, finite element calculations of ten impacts have been conducted using the quasi-static model developed in this paper for these two impact energies. The numerical results are then compared to the experimental results in Fig. 11. It shows a very good agreement which confirms the right choice of the values of $K$ and $n$.

The stress-strain curve obtained with this method corresponds to a local behavior of the surface, which means that the modifications induced by the different surface preparations and treatments are taken into account. To illustrate this difference, the stress-strain curve obtained at low strain rates and the one resulting from Hopkinson's bar testing (Bulk behavior [3]) are also plotted in Fig. 10. It points out the need to identify appropriate surface stressstrain curves when the surface behavior is concerned.

\subsection{Application to AISI316L}

The same procedure has been used with the AISI316L stainless steel. The impact energies used here are 8 and $21 \mathrm{~mJ}$. Here again the stress-strain curves identified with these two impact energy levels are very close (Fig. 12). The stress-strain curve obtained at low strain rates and the one resulting from Hopkinson's bar testing (Bulk behavior [34]) are also plotted in Fig. 12. Contrary to the AISI1045 steel the stress levels resulting from Hopkinson' bar testing and those

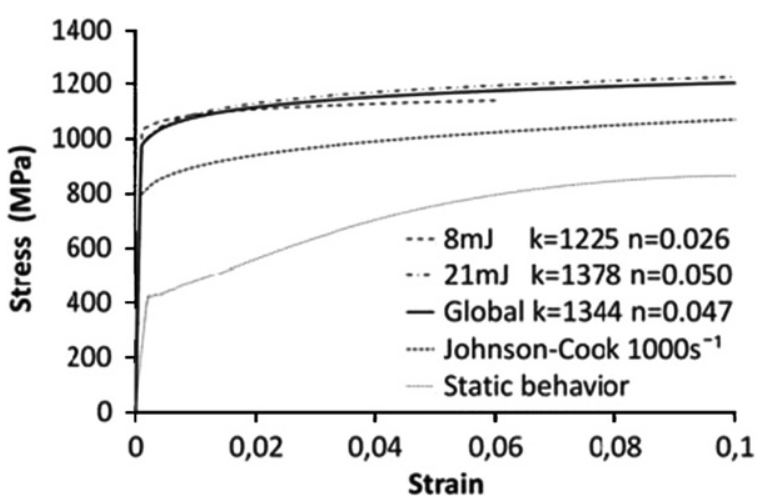

Fig. 10. Stress-strain curves identified for the AISI1045 at two impact energy levels ( 8 and $21 \mathrm{~mJ}$ ) with the present method. Comparison with the stress-strain curves resulting from Hopkinson's bar testing and quasi-static tensile testing.

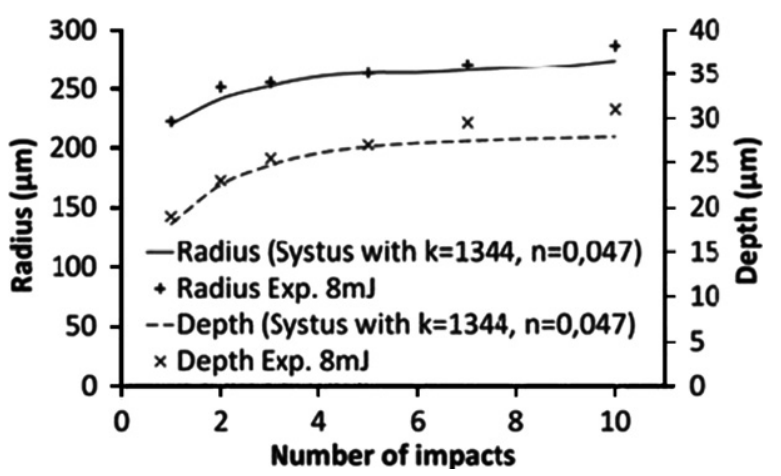

Fig. 11. Comparison of radius of the residual imprint obtained experimentally at $8 \mathrm{~mJ}$ on AISI1045 and simulated on Systus with $k=1344 \mathrm{MPa}$ and $n=0.047$.

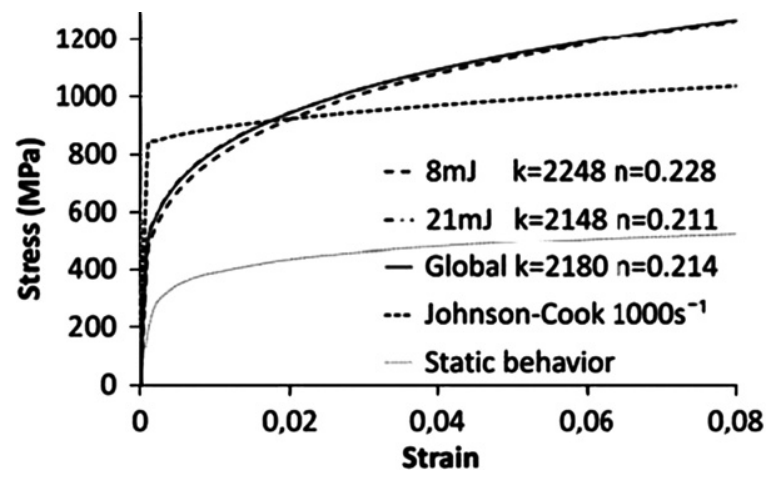

Fig. 12. Stress-strain curves identified for the AISI316L at two impact energy levels ( 8 and $21 \mathrm{~mJ}$ ) with the present method. Comparison with the stress-strain curves resulting from Hopkinson's bar testing and quasi-static tensile testing.

resulting from the proposed method are not very different. However, it appears that the strain hardening is significant for repeated impacts while there is barely any for the bulk high strain rate test. Several phenomena can be the origin of such a difference. For instance, it can be due to residual stresses or microstructure gradients. It is important to note that the main aim of this paper is to present a new material characterization method. Such kind of questions will be addressed in future works which will deal with the analysis of the response of different materials to such loadings.

\section{Conclusions}

The aim of this paper is to propose a new technique based on local micro-impact testing to characterize the stress-strain curves of metals. Therefore, a small volume of material is loaded with a strain rate [100-1000 $\mathrm{s}^{-1}$ ] close to those induced by several surface mechanical treatments. One strong interest of this method lies in its simplicity and its low cost compared to other kind of mechanical characterization such as dynamic indentation testing, where both load and displacement are measured continuously during the test. Even if the accuracy level of this last technique is better, the method presented in this paper appears to be the best compromise between precision, cost and rapidity in view of understanding the effect of surface treatments on surface mechanical properties at high strain rates. This method can be used on any kind of metals but only if the loaded volume can be considered as homogeneous. Consequently this work needs to be extended to the identification of local properties of graded materials. Future works will focus on the effect of temperature on local mechanical properties. This last point is of great interest for some manufacturing processes such as machining or finishing, where small volume are submitted to high strain rates and high temperature rises.

\section{Acknowledgments}

The authors wish to thank Ph. Gilles, D. Hertz and V. Robin from Areva-NP for their fruitful discussions and also S. Lamri for his help on the impact testing device.

\section{Appendix A. Validation of the quasi-static model}

The purpose of this section is to compare the results obtained with a dynamic explicit FEM with those obtained with the quasi-static implicit FEM described in this paper. Non-linear dynamic explicit simulations have been performed with Abaqus Explicit [35] using axisymmetric elements. Similarly to the quasi-static model, the mesh 

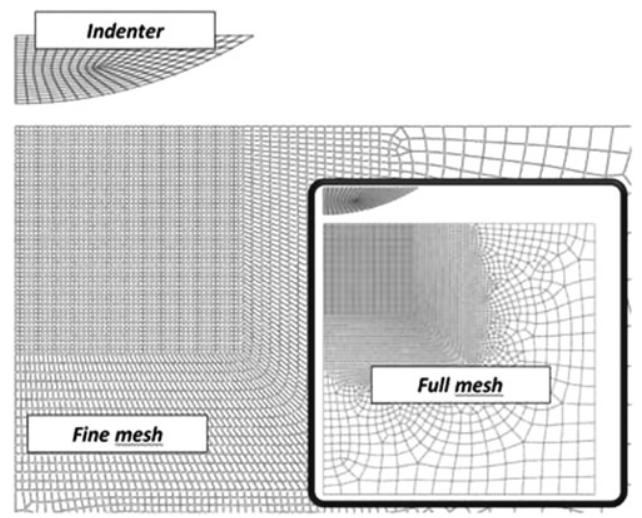

Fig. 13. Finite element mesh of the dynamic explicit FE analysis.

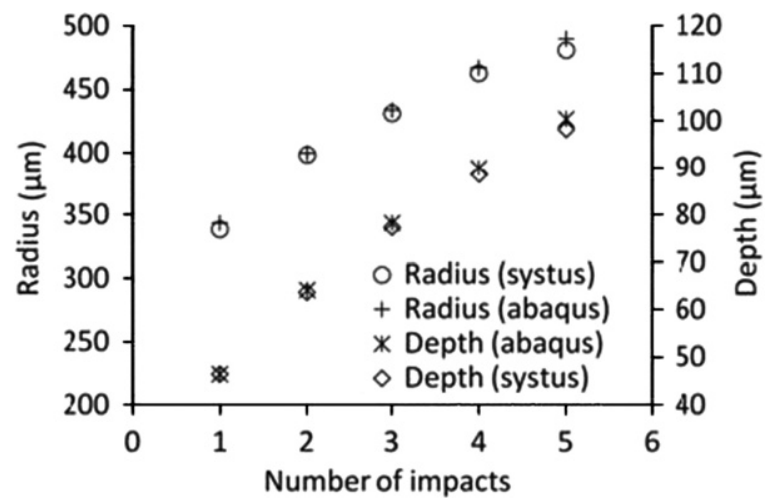

Fig. 14. Comparison of results (depth and radius of the residual imprint) obtained with a quasi-static implicit FE calculation (Systus [30]) and a dynamic explicit FE calculation (Abaqus Explicit [35]).

has been specifically refined near the contact zone (Fig. 13), and the sample is also sufficiently wide to approximate a semi-infinite solid. The mesh consists of quadrilateral elements with reduced integration and hourglass control. Contrary to quasi-static calculations, the displacement of the ball depends on its initial speed and is not monitored during the impact.

The work-piece is an elastic-plastic solid with a linear strain hardening (300 MPa at $0 \%$ plastic strain and $800 \mathrm{MPa}$ at $100 \%$ plastic strain). Ball radius is $1 \mathrm{~mm}$ and the contact is frictionless. Five balls impact the sample at $310 \mathrm{~mm} \mathrm{~s}^{-1}$, which corresponds to an impact energy of $8 \mathrm{~mJ}$. The resulting growths of depth and radius of the residual imprint are plotted in Fig. 14. It is shown that during the five impacts - i.e. the five loading-unloading cycles - the depth and radius of the residual imprint are almost similar. This comparison shows that it is possible to simulate repeated normal impacts using a quasi-static approach with enough accuracy. Let us however note that such approach is suitable because $n$ the present case study, according to Eq. (4) the impact velocity is low enough to neglect elastic wave propagations [15].

\section{Appendix B. Influence of friction}

The purpose of these calculations is to state on the effect of friction conditions on the residual impact morphology. Finite element calculations of micro-impact tests have been performed with a rough contact condition (infinite friction coefficient) and a frictionless contact condition between the indenter and the work piece. The incident impact energy used was $8 \mathrm{~mJ}$. The work-piece is an elastic-plastic solid with a linear strain hardening (300 MPa

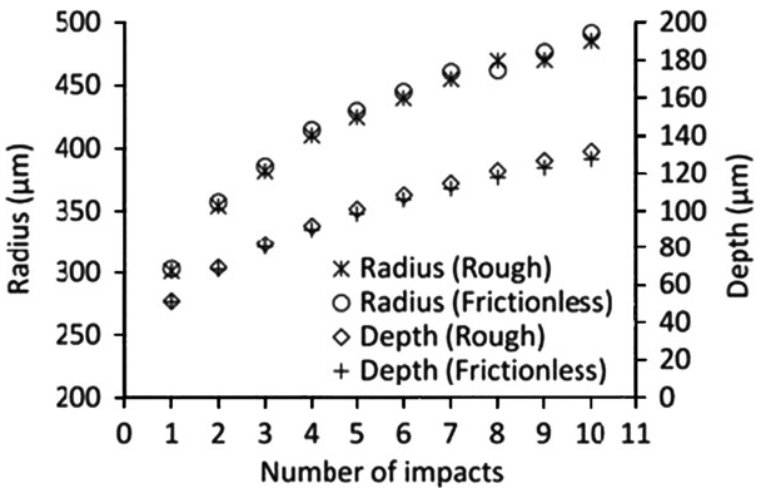

Fig. 15. Effect of friction conditions on impact crater morphology.

at $0 \%$ plastic strain and $800 \mathrm{MPa}$ at $100 \%$ plastic strain). These mechanical properties have been chosen in order to induce a high enough increase in the radius of the residual imprint at each impact. Therefore, this should highlight the effect of friction on the results [32]. The depth and radius of the residual imprint are compared in Fig. 15. From the results of these calculations, it can be concluded that the sensitivity of the identification method to the friction conditions is very weak. This is the reason why all the calculations have been performed using a frictionless contact.

\section{References}

[1] M. Barge, H. Hamdi, J. Rech, J.-M. Bergheau, J. Mater. Process. Technol. 164-165 (2005) 1148-1153.

[2] S.P.F.C. Jaspers, J.H. Dautzenbergb, J. Mater. Process. Technol. 191 (2007) $270-273$.

[3] S.P.F.C. Jaspers, J.H. Dautzenberg, J. Mater. Process. Technol. 122 (2002) $322-330$

[4] G. Kermouche, J. Rech, H. Hamdi, J.M. Bergheau, Wear 269 (2010) 86-92.

[5] W.-C. Oliver, G. Pharr, J. Mater. Res. 7 (1992) 1564-1583.

[6] G. Kermouche, J.-L. Loubet, J.-M. Bergheau, C. R. Mec. 333 (2005) 389-395.

[7] G. Kermouche, J.L. Loubet, J.M. Bergheau, Mech. Mater. 40 (2008) 271-283.

[8] Y. Tirupataiah, G. Sundararajan, J. Mech. Phys. Solids 39 (1991) 243-271.

[9] G. Subhash, B.J. Koeppel, A. Chandra, J. Eng. Mater. Technol. Trans. ASME 121 (1999) 257-263.

[10] G. Sundararajan, Y. Tirupataiah, Acta Mater. 54 (2006) 565-575.

[11] G. Sundararajan, Y. Tirupataiah, Acta Mater. 54 (2006) 577-586.

[12] A.C. Sekkal, C. Langlade, A.B. Vannes, Mater. Sci. Eng. A 393 (2005) 140-146.

[13] S. Lamri, C. Langlade, G. Kermouche, V. Martinez, Mater. Sci. Eng. A 527 (2010) 7912-7919.

[14] D. Tabor, Proc. R. Soc. Lond. A 192 (1948) 247

[15] K.L. Johnson, Contact Mechanics, Cambridge University Press, 1985.

[16] Y.T. Cheng, C.M. Cheng, Mater. Sci. Eng. R 44 (2004) 91-149.

[17] D. Tabor, J. Appl. Phys. 145 (1971) 179.

[18] H. Lee, J.H. Lee, G.M. Pharr, J. Mech. Phys. Solids 53 (2005) 2037-2069.

[19] S. Kucharski, Z. Mróz, Mater. Sci. Eng. A 318 (2001) 65-76.

[20] Y-P. Cao, J. Lu, Acta Mater. 52 (2004) 4023-4032.

[21] E-G. Herbert, G-M. Pharr, W-C. Oliver, B-N. Lucas, J-L. Hay, Thin Solid Films 398-399 (2001) 331-335.

[22] C.H. Mok, J. Duffy, Int. J. Mech. Sci. 7 (1965) 355-371.

[23] Y. Tirupataiah, G. Sundararajan, Mater. Sci. Eng. A 189 (1994) 117-127.

[24] G. Kermouche, G. Pacquaut, C. Langlade, J.M. Bergheau, C. R. Mec. 339 (2011) 552-562.

[25] S.A. Meguid, G. Shagal, J.C. Stranart, Int. J. Impact Eng. 24 (2002) 119-134.

[26] K. Dai, L. Shaw, Mater. Sci. Eng. A 463 (2007) 45-53.

[27] G.H. Majzoobi, R. Azizi, A. Alavi Nia, J. Mater. Process. Technol. 164-165 (2005) 1226-1234.

[28] M. Frija, T. Hassine, R. Fathallah, C. Bouraoui, A. Dogui, Mater. Sci. Eng. A 426 (2006) 173-180

[29] J.P. Nobre, A.M. Dias, R. Gras, Resistance of a ductile steel surface to spherical normal impact indentation: use of a pendulum machine, Wear 211 (1997) $226-236$.

[30] ESI Group, Systus/Sysweld. Users Manual, 2004.

[31] M. Dao, K. Chollacoop, K.J. Van Vliet, T.A. Venkatesh, S. Suresh, Acta Mater. 49 (2001) 3899-3918.

[32] J.L. Bucaille, S. Stauss, E. Felder, J. Michler, Acta Mater. 51 (2003) 1663-1678.

[33] J.M. Collin, T. Parenteau, G. Mauvoisin, P. Pilvin, Comput. Mater. Sci. 46 (2009) 333-338.

[34] D. Umbrello, R. M'Saoubi, J.C. Outeiro, Int. J. Mach. Tools Manuf. 47 (2007) 462-470.

[35] Dassault Systems, Abaqus Explicit, Users Manual, 2012. 